\title{
Contribution of myeloperoxidase and inducible nitric oxide synthase to pathogenesis of psoriasis
}

\author{
Nursel Dilek¹, Aziz Ramazan Dilek², Yakup Taşkın³, Taşkın Erkinüresin ${ }^{3}$, Ömer Yalçın³, Yunus Saral ${ }^{1}$ \\ 1Dermatology Department, Recep Tayyip Erdoğan University, Medical Faculty Hospital, Rize, Turkey \\ ${ }^{2}$ Microbiology Department, Recep Tayyip Erdoğan University, Medical Faculty Hospital, Rize, Turkey
} ${ }^{3}$ Pathology Department, Şevket Yılmaz Education and Research Hospital, Bursa, Turkey

Adv Dermatol Allergol 2016; XXXIII (6): 435-439

DOI: 10.5114/ada.2016.63882

\begin{abstract}
Introduction: Histological changes of psoriasis include invasion of neutrophils into the epidermis and formation of Munro abscesses in the epidermis. Neutrophils are the predominant white blood cells in circulation when stimulated; they discharge the abundant myeloperoxidase (MPO) enzyme that uses hydrogen peroxide to oxidize chloride for killing ingested bacteria.

Aim: To investigate the contribution of neutrophils to the pathogenesis of psoriasis at the blood and tissue levels through inducible nitric oxide synthase (iNOS) and MPO.

Material and methods: A total of 50 adult patients with a chronic plaque form of psoriasis and 25 healthy controls were enrolled to this study. Serum MPO and iNOS levels were measured using ELISA method. Two biopsy specimens were taken in each patient from the center of the lesion and uninvolved skin. Immunohistochemistry was performed for MPO and iNOS on both normal and psoriasis vulgaris biopsies.

Results: While a significant difference between serum myeloperoxidase levels were detected, a similar statistical difference between participants in the serum iNOS levels was not found. In immunohistochemistry, intensely stained leukocytes with MPO and intensely staining with iNOS in psoriatic skin was observed.

Conclusions: Neutrophils in psoriasis lesions are actively producing MPO and this indirectly triggers the synthesis of iNOS. Targeting of MPO or synthesis of MPO in the lesion area may contribute to development of a new treatment option.
\end{abstract}

Key words: psoriasis, myeloperoxidase, inducible nitric oxide.

\section{Introduction}

The inflammatory infiltrate in the psoriatic skin is characterized immunohistochemically by different subsets of immunocompetent cells such as T-lymphocytes, monocytes, macrophages, mast cells and polymorphonuclear leukocytes (PMN) [1]. In a certain time, it has been widely recognized that the interaction of $T$ cells and dendritic cells (DCS) creates a type 1 inflammatory environment by secreting large amounts of Th1 type cytokines, leading to the development of psoriasis. Whereas, recently obtained findings suggest that psoriasis cannot be simply defined as one subset of Th cells-mediated disease. Instead, all these pathogenic Th cells are implicated in the disease development, which interact with other types of T cells, DCs and neutrophils to create a chronic inflammatory environment for the maintenance of pso- riatic plaque [2]. The early histological changes seen in a psoriatic lesion include invasion of neutrophils into the epidermis and formation of Munro abscesses in the epidermis [3]. In the story of neutrophils collection in the inflammation area, adhesion to endothelial cells (ECS) as a critical process in the extravasation of neutrophils into inflamed perivascular tissue [4]. Enhanced adhesion and migration into the tissue involves up regulation and activation of CD11b/CD18 on neutrophils and intercellular adhesion molecule 1 (ICAM-1) on endothelial cells [3] Clinical importance of neutrophils for the pathogenesis of psoriasis was reported by Toichi et al. In this report, psoriasis began to improve rapidly when peripheral blood neutrophil counts decreased to several hundred per micro liter in a case of drug-induced agranulocytosis who has psoriasis. In the patient no new psoriatic plaques

Address for correspondence: Nursel Dilek, Dermatology Department, Recep Tayyip Erdoğan University, Medical Faculty Hospital, 53000 Rize, Turkey, phone/fax: +90 464 2130491, e-mail: nur.dilek@hotmail.com

Received: 4.11.2015, accepted: 27.11.2015. 
were observed until neutrophil counts increased to several thousand per micro liter [5]. Neutrophils are the predominant white blood cells in circulation and when stimulated, neutrophils consume oxygen in a respiratory burst that produces superoxide and hydrogen peroxide and simultaneously discharge the abundant heme enzyme myeloperoxidase (MPO) that uses hydrogen peroxide to oxidize chloride, bromide and thiocyanate to the respective hypohalous acids and hypothiocyanite. These oxidants kill ingested bacteria but are also implicated in tissue damage [6]. But studies have shown that the story of MPO is not limited with this, MPO binds to CD11b/CD18 integrins on PMNs, leading to induction of intracellular signaling cascades and translating into upregulated PMN degranulation, CD11b surface expression, and NADPH oxidase activity in an autocrine manner [7].

\section{Aim}

In the present study, we aimed to investigate the contribution of neutrophils to the pathogenesis of psoriasis through inducible nitric oxide synthase (iNOS) and MPO which are believed to have a relationship with each other at the blood and tissue levels in patients with psoriasis [8]

\section{Material and methods}

A total of 50 adult patients (27 males and 23 females, mean age: $36.8 \pm 8.2$ ) with a chronic plaque form of psoriasis and 25 healthy controls (14 males and 10 females, mean age: $36.9 \pm 8.2$ ) were enrolled to this study. The patients were divided in two groups: the first group consisted of 25 patients with a high Psoriasis Area and Severity Index (PASI) score (PASI score $\geq 7$, mean PASI score: $9.66 \pm 2.41$ ) and the second group covered 25 patients with a mild PASI score (PASI score $\leq 7$, mean PASI score: $2.57 \pm 1.23)$. The demographic and clinical characteristics of participants are presented in Table 1.

The patients who have an additional disease such as infection, cancer and autoimmune diseases or received systemic therapy recently (for the last 15 days) were excluded from the study. The PASI was performed for clinical evaluation of disease severity before taking blood samples. Complete blood cell count of participants was tested with the cell counter system (Abbott, Cell-Dyn 3700) ac- cording to the manufacturer's instructions. Serum of all participants was immediately obtained by centrifugation, transferred into cryotubes, and stored at $-70^{\circ} \mathrm{C}$ until assayed. Serum myeloperoxidase levels were measured using ELISA method (Human MPO Instant ELISA kit, eBioscience, Austria) according to the manufacturer's protocol. Serum iNOS levels were measured using ELISA method (Human inducible nitric oxide synthase (iNOS) ELISA Kit, Cusabio, Wuhan, China) according to the manufacturer's protocol. Absorbance (OD) of each well determined at $450 \mathrm{~nm}$ with a microtiter plate reader (Multiskan GO, Thermo Scientific, Waltham, MA, USA) in the $5^{\text {th }}$ min. Standard curves were fitted using Titri ELISA software. The fitted curves were then used to convert sample absorbance readings to MPO and iNOS concentration.

After infiltration with a local anesthetic, two biopsy specimens were taken. In each patient a $6 \mathrm{~mm}$ punch biopsy was taken from the center of the lesion. Another $6 \mathrm{~mm}$ punch biopsy was taken from uninvolved skin, which was at least $10 \mathrm{~cm}$ apart from any psoriatic plaque. Immunohistochemistry was performed on both normal and psoriasis vulgaris biopsies of deparaffinized human skin tissue. To expose target proteins, heat-induced antigen retrieval was performed using $10 \mathrm{mM}$ sodium citrate $(\mathrm{pH}$ = 6.0) buffer, microwaved for 8-15 min. Following antigen retrieval, tissues were blocked in 3\% BSA-PBS for 30 minutes at room temperature. Tissues were then probed at a dilution of $1 / 200$ with a rabbit polyclonal antibody recognizing iNOS (ab3523-abcam) and at a dilution of 1/50 with a Rabbit polyclonal Anti-Myeloperoxidase antibody (ab45977-abcam) or without the primary antibody (negative control) overnight at $4^{\circ} \mathrm{C}$ in a humidified chamber. Tissues were washed extensively with PBST (Phosphate buffered saline-Triton X-100) and endogenous peroxidase activity was quenched with a peroxidase suppressor. Detection was performed using a biotin conjugated secondary antibody and SA-HRP (Streptavidin-horseradish peroxidase), followed by colorimetric detection using DAB (3,3'-diaminobenzidine). Tissues were counterstained with hematoxylin and prepped for mounting.

\section{Results}

According to the obtained results, a statistically significant difference in terms of the number of neutrophils

Table 1. The demographic and clinical characteristics of participants

\begin{tabular}{|c|c|c|c|}
\hline Parameter & High PASI score & Mild PASI score & Healthy controls \\
\hline Subjects & 25 & 25 & 25 \\
\hline Mean age & $36.20 \pm 7.76$ & $37.48 \pm 8.78$ & $36.92 \pm 8.28$ \\
\hline Male/female & $15 / 10$ & $12 / 13$ & $14 / 11$ \\
\hline Lesion type & Plaque form & Plaque form & - \\
\hline PASI score & $9.66 \pm 2.41$ & $2.57 \pm 1.23$ & - \\
\hline
\end{tabular}


Table 2. Serum myeloperoxidase levels (ANOVA)

\begin{tabular}{lcccc}
\hline Variable & Sum of squares & $\mathrm{d} f$ & Mean square & $\boldsymbol{F}$ \\
\hline Between groups & $3.182 \mathrm{E} 7$ & 2 & $1.591 \mathrm{E} 7$ & 73.820 \\
\hline Within groups & $1.552 \mathrm{E} 7$ & 72 & 215554.910 & 0.000 \\
\hline Total & $4.734 \mathrm{E} 7$ & 74 & & \\
\hline
\end{tabular}

in the blood of the participants was not available $(p>$ 0.05). In the statistical examination, a significant difference between serum myeloperoxidase levels was detected ( $p<0.05$, ANOVA test, Table 2). Serum myeloperoxidase levels in a high PASI group were found to be significantly higher than the others (Figure 1). However, a similar statistical difference between participants in the serum iNOS levels was not found (ANOVA test, Table 3 and Figure 2). When immunostaining was performed to assess the relationship between iNOS and MPO locally, intensely stained leukocytes with MPO were seen in psoriatic skin. Similarly, intensely staining with iNOS in psoriatic skin was observed. Comparison of immunostaining in the normal skin and lesional skin is shown in Figure 3. These results are surprisingly different from the serum results obtained using with ELISA method.

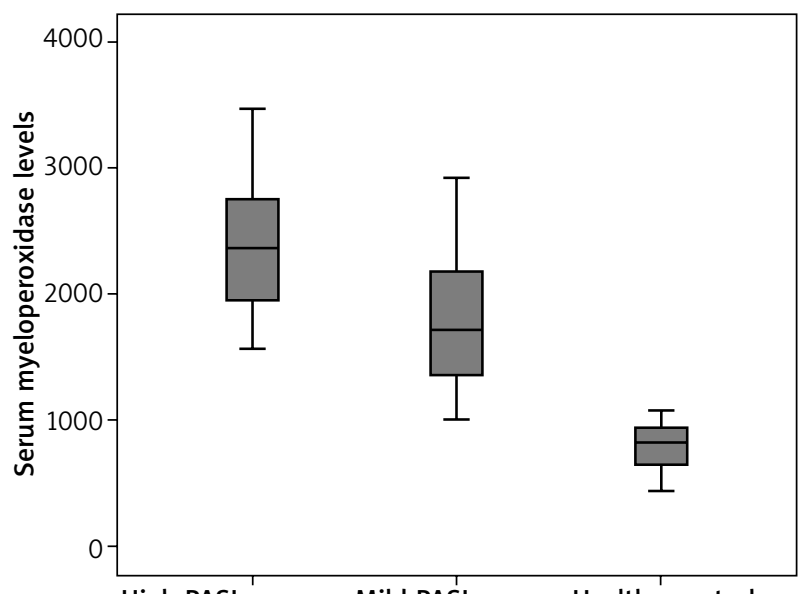

High PASI score Mild PASI score Healthy controls

Figure 1. Serum myeloperoxidase levels of participants in Boxplot

Table 3. Serum iNOS levels (ANOVA)

\begin{tabular}{|c|c|c|c|c|c|}
\hline Variable & Sum of squares & $\mathrm{d} f$ & Mean square & $F$ & Sig. \\
\hline Between groups & 14,804 & 2 & 7.402 & 1.693 & 0.191 \\
\hline Within groups & 314,764 & 72 & 4.372 & & \\
\hline Total & 329,568 & 74 & & & \\
\hline
\end{tabular}

\section{Discussion}

Psoriatic lesions are characterized by a focal dense infiltration of neutrophils, T lymphocytes and macrophages that migrate from the vascular to the dermal compartment and through all layers of the epidermis up to the stratum corneum [9]. Neutrophils, which are a type of polymorphonuclear leukocyte, are well recognized as one of the major players during acute inflammation. However, recent data have shown that neutrophil functions extend beyond their roles in acute infection. Despite their short life, during inflammation neutrophils become activated and their longevity increases several folds, which ensures the presence of primed neutrophils at the site of inflammation. A longer lifespan may allow neutrophils to carry out more complex activities in a tissue, such as contributing to the resolution of inflammation or shaping adaptive immune responses [10]. Neutrophils are equipped with an arsenal of antimicrobial proteins including reactive oxygen species-producing enzymes, chelators of vitamins and trace elements and enzymes

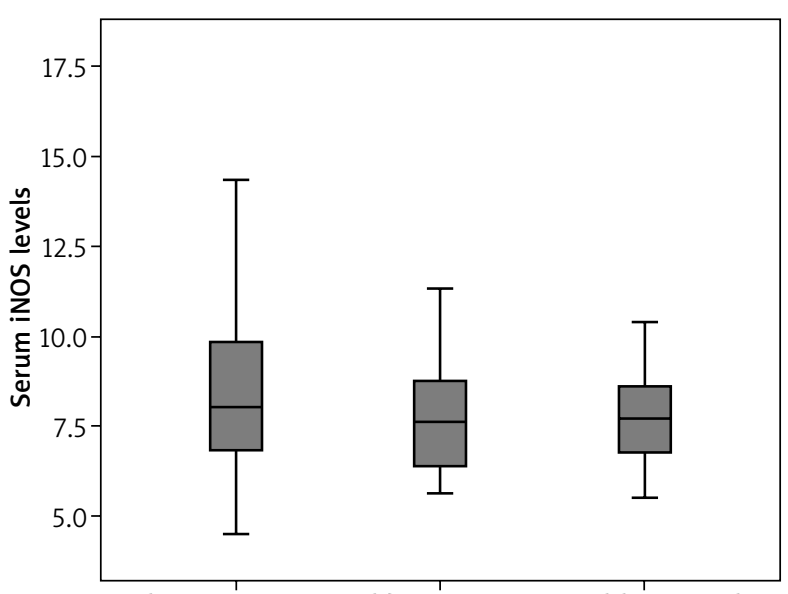

High PASI score Mild PASI score Healthy controls

Figure 2. Serum iNOS levels of participants in Boxplot 

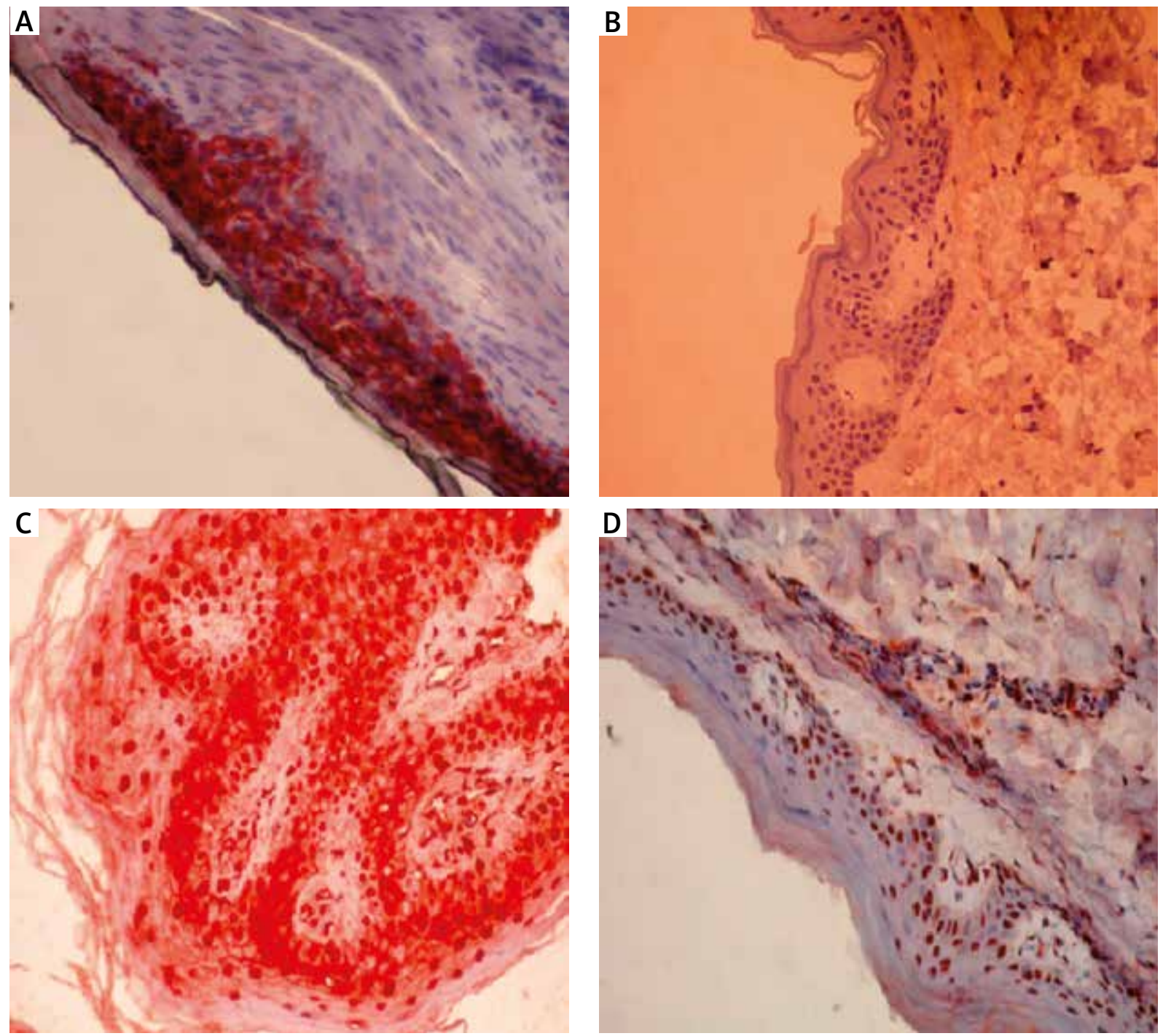

Figure 3. A - Intensely stained leukocytes with MPO were seen in the psoriatic skin, B - staining of the normal skin with $\mathrm{MPO}, \mathrm{C}$ - intensely staining with iNOS in the psoriatic skin, D - staining of the normal skin with iNOS

capable of degrading microbial proteins or cell wall components. That weaponry may not only be detrimental to microbes but it can also damage components of the host tissues [11]. One of the major weaponry of neutrophils is enzyme MPO (MPO accounts for 5\% of total neutrophil proteins and is a major granule constituent), which in combination with $\mathrm{H}_{2} \mathrm{O}_{2}$ and chloride, constitutes a potent antimicrobial system [12]. Selectively activation of neutrophils in psoriasis was shown in a previous study by Lundin et al. [13]. Likewise significantly increased MPO levels which showed a positive correlation with disease severity in our study refers to a specifically local and systemic neutrophil activation. Another study supporting this was made by Lau et al. and showed that MPO binds to CD11b/CD18 integrins on PMNs, leading to induction of intracellular signaling cascades and translating into up-regulated PMN degranulation [7]. This finding helps to explain the excess production of MPO in psoriatic lesions.
Nitric oxide synthases are hemoproteins that catalyze oxidation of L-arginine to nitric oxide (NO) and L-citrulline. Nitric oxide is a ubiquitous free radical, and it plays an important role either as a messenger or as a destructive molecule in inflammation and has been shown to modulate the inflammatory process [14]. Inducible NO synthase is only expressed after cell activation through variety of inflammatory mediators such as cytokines, lipopolysaccharide (LPS) and others and then produces NO for comparatively long periods of time (hours to days) in contrast to other NO synthase. Thus, regulated short pulsative synthesis versus constant NO production differentiates between physiological and pathophysiological actions of NO [15]. Our findings obtained in this study indicate local activation of iNOS synthase. This was shown in studies; iNOS catalytic activity is down regulated by NO feedback inhibition by a mechanism that constrains the enzyme to operate at only a fraction of its maximum 
activity and mammalian peroxidases such as MPO may operate as alternative pathways for catalytic removal of $\mathrm{NO}$ at sites of inflammation [8]. These data suggest that as in our findings, increased MPO levels in the lesion may trigger the iNOS synthase indirectly. In our study, the amount of both enzyme MPO and iNOS was found to be increased in the lesion correlated with the clinical severity.

In addition, recently conducted studies showed that the blood neutrophil counts are increasing in patients with psoriasis and amount of superoxide anions are increased in these neutrophils $[16,17]$.

Consequently, based on the findings obtained from the study, neutrophils in psoriasis lesions are actively producing MPO and this indirectly triggers the synthesis of iNOS and consists of an autonomous structure. If possible, the synthesis of molecules which target MPO or synthesis of MPO in the lesion area may contribute to the deterioration of this autonomous structure and development of a new treatment option.

\section{Conflict of interest}

The authors declare no conflict of interest.

\section{References}

1. van Pelt JP, Kuijpers SH, van de Kerkhof PC, et al. The CD11b/ CD18-integrin in the pathogenesis of psoriasis. J Dermatol Sci 1998; 16: 135-43.

2. Cai Y, Fleming C, Yan J. New insights of T cells in the pathogenesis of psoriasis. Cell Mol Immunol 2012; 9: 302-9.

3. Sjögren F, Ljunghusen O, Baas A, et al. Expression and function of beta 2 integrin CD11B/CD18 on leukocytes from patients with psoriasis. Acta Derm Venereol 1999; 79: 105-10.

4. Wetzel A, Wetzig T, Haustein UF, et al. Increased neutrophil adherence in psoriasis: role of the human endothelial cell receptor Thy-1 (CD90). J Invest Dermatol 2006; 126: 441-52.

5. Toichi E, Tachibana T, Furukawa F. Rapid improvement of psoriasis vulgaris during drug-induced agranulocytosis. Am Acad Dermatol 2000; 43: 391-5.

6. Paumann-Page M, Furtmüller PG, Hofbauer S, et al. Inactivation of human myeloperoxidase by hydrogen peroxide. Arch Biochem Biophys 2013; 539: 51-62.

7. Lau D, Mollnau H, Eiserich JP, et al. Myeloperoxidase mediates neutrophil activation by association with CD11b/CD18 integrins. Proc Natl Acad Sci USA 2005; 102: 431-6.

8. Galijasevic S, Saed GM, Diamond MP, et al. Myeloperoxidase up-regulates the catalytic activity of inducible nitric oxide synthase by preventing nitric oxide feedback inhibition. Proc Natl Acad Sci USA 2003; 100: 14766-71.

9. Pietrzak A, Kozioł-Montewka M, Lecewicz-Toruń B, et al. Is there any correlation between the total number of neutrophils in plasma and concentration of interleukin-8 in psoriatic patients? Med Sci Monit 2000; 6: 867-70.

10. Kolaczkowska E, Kubes P. Neutrophil recruitment and function in health and inflammation. Nat Rev Immunol 2013; 13: 159-75.

11. Németh T, Mócsai A. The role of neutrophils in autoimmune diseases. Immunol Lett 2012; 143: 9-19.
12. Klebanoff SJ, Kettle AJ, Rosen H, et al. Myeloperoxidase: a front-line defender against phagocytosed microorganisms. J Leukoc Biol 2013; 93: 185-98.

13. Lundin A, Håkansson L, Hällgren R, et al. Increased in vivo secretory activity of neutrophil granulocytes in patients with psoriasis and palmoplantar pustulosis. Arch Dermatol Res 1985; 277: 179-84.

14. Hazam RK, Deka M, Kar P. Role of nitric oxide synthase genes in hepatitis E virus infection. J Viral Hepat 2014; 21: 671-9.

15. Kröncke KD, Fehsel K, Kolb-Bachofen V. Inducible nitric oxide synthase in human diseases. Clin Exp Immunol 1998; 113: 147-56.

16. Kim DS, Shin D, Lee MS, et al. Assessments of neutrophil to lymphocyte ratio and platelet to lymphocyte ratio in Korean patients with psoriasis vulgaris and psoriatic arthritis. J Dermatol 2016; 43: 305-10.

17. Guérard S, Allaeys I, Martin G, et al. Psoriatic keratinocytes prime neutrophils for an overproduction of superoxide anions. Arch Dermatol Res 2013; 305: 879-89. 